\title{
Lithium Adsorption on Zigzag Graphene Nanoribbons
}

\author{
Chananate Uthaisar, Veronica Barone * * and Juan E. Peralta \\ Department of Physics, Central Michigan University \\ Mount Pleasant, MI 48859.
}

(Dated: October 27, 2018)

\begin{abstract}
We have studied the adsorption of Li atoms at the hollow sites of graphene nanoribbons (zigzag and armchair), graphene, and fullerenes by means of density functional theory calculations including local and semilocal functionals. The binding energy of a Li atom on armchair nanoribbons (of about $1.70 \mathrm{eV}$ for LSDA and $1.20 \mathrm{eV}$ for PBE) is comparable to the corresponding value in graphene (1.55 and $1.04 \mathrm{eV}$ for LSDA and PBE, respectively). Notably, the interaction between Li and zigzag nanoribbons is much stronger. The binding energy of $\mathrm{Li}$ at the edges of zigzag nanoribbons is about $50 \%$ stronger than in graphene for the functionals studied here. While the charge transfer between the $\mathrm{Li}$ adatom and the zigzag nanoribbon significantly affects the magnetic properties of the latter providing an additional interaction mechanism that is not present in two-dimensional graphene or armchair nanoribbons, we find that the morphology of the edges, rather than magnetism, is responsible for the enhanced Li-nanoribbon interaction.

PACS numbers: Valid PACS appear here
\end{abstract}

\section{INTRODUCTION}

The recent experimental realization of atomically thin, long, and narrow strips of graphene (graphene nanoribbons, GNRs) [1, 2, 3] has sparked an intense research effort toward the understanding of these novel materials. Experimental evidence of ballistic electronic transport, large phase coherence lengths, and current density sustainability, 1, 2] exotic magnetic properties, 3, [4, 5, 6, 7, 8, 9, 10, 11, 12] quasi-relativistic behavior, 13, 14, 15, 16, 17 and electronic structure engineering capabilities 10, 18, 19, 20, 21, 22, 23, 24, 25] identify low-dimensional graphene as one of the most promising materials for novel electronic and spintronic devices. Unlike two-dimensional graphene, GNRs present electronic confinement in the transverse direction giving rise to peculiar electronic properties, similar to the case of single walled carbon nanotubes (SWNTs). As in SWNTs, these properties depend strongly on the crystallographic orientation of their main axis, i.e. armchair or zigzag. In addition to their remarkable electronic and magnetic properties, GNRs present a distinctive chemistry due to the presence of reactive edges. 25, 26, 27]

The interaction between $\mathrm{Li}$ atoms and carbon-based materials has been a subject of intensive study motivated by their potential for more efficient Li-ion batteries and hydrogen storage media. While the detailed interaction mechanism is still controversial, [28, 29] it is commonly accepted that it presents a mostly ionic character with a substantial electronic charge transfer from the $\mathrm{Li}$ atom to the graphitic surface. [30, 31, 32, 33] In a recent work, Yoo et al. presented experimental evidence of a higher Li storage capacity in graphene with respect to graphite. [30] Li adsorption on fullerenes has been studied

*Electronic address: v.barone@cmich.edu theoretically by Sun et al. 32] In that work the authors found that $\mathrm{Li}_{12} \mathrm{C}_{60}$ can store a high density of molecular hydrogen. Recently, Ataca et al. presented a theoretical study of the adsorption of $\mathrm{Li}$ atoms on graphene with notably large Li densities. 34] However, the binding energies of $\mathrm{Li}$ atoms of such systems, with high Li coverage, are significantly lower than that of a less dense Li-graphene system due to the electrostatic repulsion between $\mathrm{Li}$ ions.

In view of all the recent focus on Li storage in lowdimensional carbon materials, we present this work with the aim of studying the adsorption mechanisms and binding energies of $\mathrm{Li}$ atoms on graphene nanoribbons and assess their Li intake capacity compared to other low-dimensional carbon materials. In what follows, we present theoretical evidence, based on density functional theory calculations, of a stronger interaction between $\mathrm{Li}$ atoms and zigzag nanoribbons in comparison with either graphene or armchair nanoribbons.

\section{METHODOLOGY}

We have carried out all calculations utilizing a development version of the Gaussian suite of programs. 35] In this program, solid state calculations are performed using all-electron Gaussian basis sets and periodic boundary conditions in one, two, or three dimensions. This flexibility allows for the treatment of low-dimensional structures, such as graphene or graphene nanoribbons, avoiding replicas in the $z$ direction. Calculations have been performed using an spin-polarized Kohn-Sham approach within the local spin density approximation (LSDA) [36, 37] and the generalized gradient approximation of Perdew et al. 38, 39] We chose the double-zetha 6-31G** basis set. [40] Despite the fact that the ionic character of the Li-grpahene interaction should be qualitatively well reproduced by either LSDA or GGA functionals, we studied the case of $\mathrm{Li}$ adsorption on zigzag nanorib- 

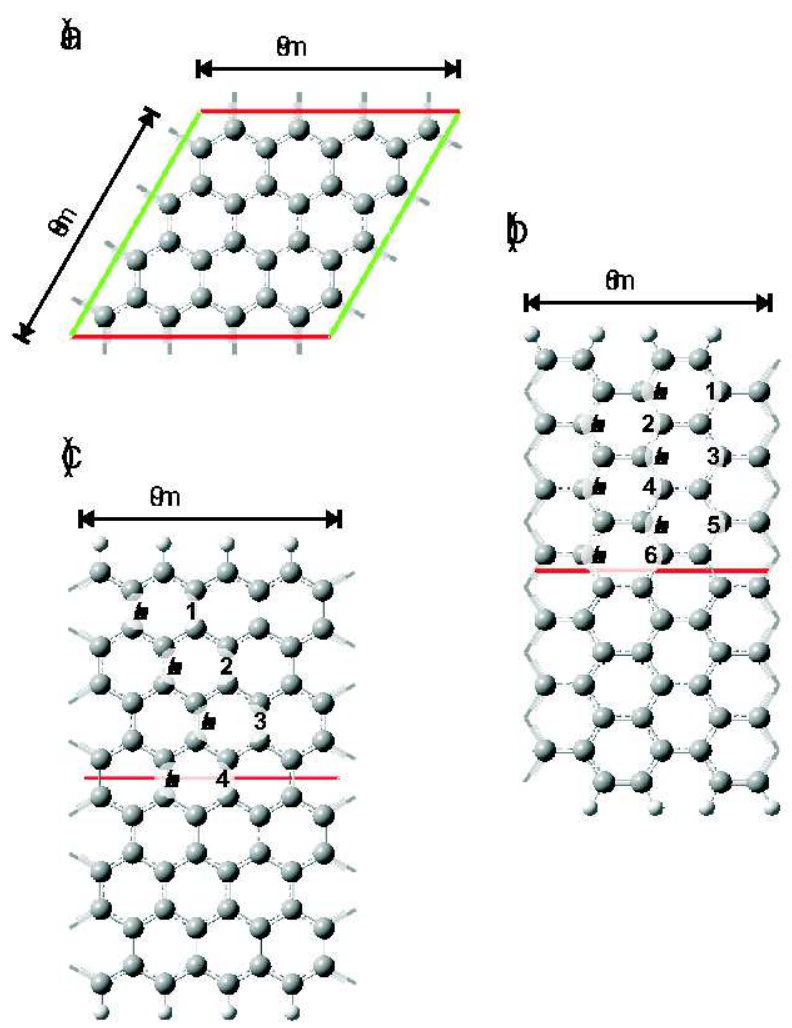

FIG. 1: Schematic diagrams of the supercells used for a) twodimensional graphene, b) armchair graphene nanoribbon, and c) zigzag graphene nanoribbon.

bons using an hybrid density functional. As upon Li adsorption the systems turn into metals, conventional hybrid functionals present convergence problems due to the portion of Hartree-Fock type of exchange included in the exchange-correlation energy. An efficient alternative to deal with this problem is the screened-exchange hybrid functional, HSE, developed by Heyd, Scuseria, and Ernzerhof. 41, 42, 43, 44]

The reciprocal space integration has been performed in a $10 \times 10$ uniform k-point grid for graphene and in a 35 uniform k-point grid for the one-dimensional nanoribbons. All the structures have been fully relaxed to account for any rearrangement in the graphitic surfaces due to the presence of the $\mathrm{Li}$ atom until the maximum and root mean square atomic forces are less than $0.02 \mathrm{eV} /$ Åand $0.015 \mathrm{eV} / \AA$, respectively, and the maximum and root mean square atomic displacements between consecutive iterations are less than $10^{-3}$ Ånd $610^{-4} \AA$, respectively.

We have studied $\mathrm{Li}$ adsorption on three representative graphene surfaces (shown in Figure 1). The unit cell for the first system consists of an isolated $(4 \times 4)$ supercell of two-dimensional graphene (without replica in the $z$ direction) with $32 \mathrm{C}$ atoms and one $\mathrm{Li}$ atom with a Li...Li distance between cells of $0.98 \mathrm{~nm}$ (Figure 1-a).
The interaction Li-graphene in this particular configuration has been studied before and therefore provides a good reference system for comparison purposes. [45, 46] The second system consists of an isolated supercell of an armchair nanoribbon $1.58 \mathrm{~nm}$ wide, containing $56 \mathrm{C}$ atoms, $8 \mathrm{H}$ atoms passivating the edges, and one $\mathrm{Li}$ atom (Figure 1-b). Within this configuration the Li....Li distance between cells is about $0.86 \mathrm{~nm}$. Finally we have chosen an isolated supercell of a zigzag nanoribbon 1.57 $\mathrm{nm}$ wide, containing $64 \mathrm{C}$ atoms, $8 \mathrm{H}$ atoms passivating the edges and one $\mathrm{Li}$ atom in such a way that the Li....Li distance between cells is $0.99 \mathrm{~nm}$ (Figure 1-c). For comparison purposes, we have also considered the case of $\mathrm{Li}$ adsorption on fullerenes, which presents one of the strongest adsorptions reported between $\mathrm{Li}$ and graphitic materials. 32.

It has been shown in several studies that alkali metal adsorption on graphitic surfaces takes place preferentially on top of the hexagon (hollow site) instead of on top of a $\mathrm{C}$ atom or on top of a C-C bond (bridge site). [45, 46] Therefore, we have studied the interaction between a $\mathrm{Li}$ atom on the hollow site of the graphitic materials in the three configurations described above and checked that the hollow sites are more stable for the case of the zigzag nanoribbon (Figure 1-c).

Since we are employing a finite localized basis set, our calculations are subject to basis set superposition error (BSSE). Although the correction for BSSE can be demanding due to the large number of configurations studied here and the different spin polarized ground states of the constituents, we can estimate its magnitude by calculating it using the counterpoise method [47] in the case of $\mathrm{Li}$ adsorbed on two-dimensional graphene. In this case, basis set truncation introduces an error in the computed binding energy smaller than $4 \%$. For this reason, we neglect the BSSE correction in the rest of this work.

\section{RESULTS}

\section{Graphene, fullerenes, and armchair nanoribbons}

Several papers appeared in the literature presenting DFT calculations in $(4 \times 4)$ graphene containing one adatom per $32 \mathrm{C}$ atoms. For instance, Khantha at al. [48] report a binding energy $\left(E_{b}\right)$ of $1.60 \mathrm{eV}$; Valencia et al. 45] report the Li binding energy in this system to be $1.55 \mathrm{eV}$ with LSDA and $1.01 \mathrm{eV}$ with the generalized gradient approximation functional of Perdew-BurkeErnzerhof, PBE, while Chan et al. report a binding energy of $1.10 \mathrm{eV}$ with PBE as well. [46] More recently, Ataca et al. calculated the binding energy for $\mathrm{Li}$ on (4x4) graphene to be $1.93 \mathrm{eV}$ with LSDA. 34] For this system, we obtain a binding energy of $1.55 \mathrm{eV}$ (LSDA) and $1.04 \mathrm{eV}$ (PBE) in good agreement with the values reported by Valencia et al.[45], Khantha at al.,[48] and Chan et al. 46.

The interaction of $\mathrm{Li}$ with $\mathrm{C}_{60}$ is stronger than with 
TABLE I: Calculated Li-hexagon distances, magnetic moments per cell and Mulliken atomic charges on the Li atom adsorbed on graphene, $C_{60}$, and the four possible configurations of $\mathrm{Li}$ on the hollow site of a zigzag GNRs.

\begin{tabular}{|c|c|c|c|c|c|c|c|c|}
\hline & \multicolumn{2}{|c|}{$d(\stackrel{\circ}{A})$} & \multicolumn{4}{|c|}{$\mu\left(\mu_{B}\right)$} & \multicolumn{2}{|c|}{ Li charge } \\
\hline & LSDA & $\overline{\mathrm{PBE}}$ & & LSDA & $\mathrm{PBE}$ & & LSDA & $\mathrm{PBE}$ \\
\hline Graphene & 1.64 & 1.70 & & 0.00 & 0.00 & & 0.36 & $\overline{0.41}$ \\
\hline$C_{60}$ & 1.70 & 1.76 & & 1.00 & 1.00 & & 0.42 & 0.39 \\
\hline & & & & & & & & \\
\hline Zigzag nanoribbon & LSDA & PBE & $\overline{\mathrm{LSDA}}$ & $\overline{\mathrm{PBE}}$ & LSDA & $\overline{\mathrm{PBE}}$ & LSDA & PBE \\
\hline hex -1 & 1.70 & 1.71 & 1.00 & 1.18 & - & - & 0.33 & 0.38 \\
\hline hex -2 & 1.70 & 1.74 & 1.42 & 1.57 & 0.59 & 0.65 & 0.39 & 0.44 \\
\hline hex -3 & 1.71 & 1.75 & 1.53 & 1.68 & 0.27 & 0.29 & 0.41 & 0.46 \\
\hline hex -4 & 1.70 & 1.75 & 1.56 & 1.71 & 0.00 & 0.00 & 0.41 & 0.46 \\
\hline
\end{tabular}

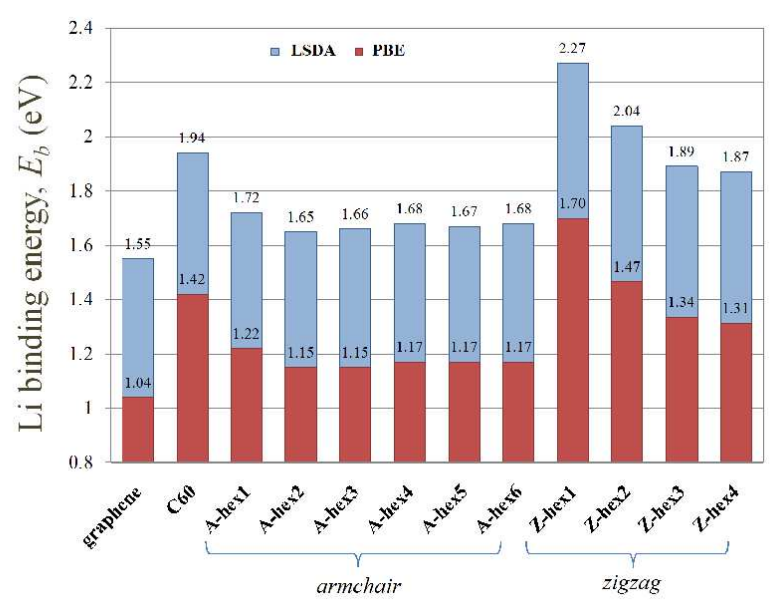

FIG. 2: LSDA and PBE Li binding energies on the hollow sites of the different systems considered in this work: $(4 \times 4)$ graphene, $C_{60}$, armchair nanoribbons with $1 \leq i \leq 6$, and zigzag nanoribbons with $1 \leq i \leq 4$.

armchair GNRs. Both hollow sites (on top of the pentagon and on top of the hexagon) are degenerate with a binding energy of $1.94 \mathrm{eV}$ for LSDA and $1.42 \mathrm{eV}$ for PBE. This value, calculated using PBE, is smaller than the $1.80 \mathrm{eV}$ obtained by Sun et al. with the PBE functional. 32.

Due to the quasi-one-dimensional nature of the nanoribbons, there are several possible distinctive configurations for $\mathrm{Li}$ on the hollow sites. These different possibilities are schematized in Figures 1-b for armchair and 1-c for zigzag nanoribbons and denoted $h e x-i$ with $1 \leq i \leq 6$ for armchairs and $1 \leq i \leq 4$ for zigzags. As shown in Figure 2, the interaction of Li with armchair nanoribbons is to some extent stronger than with graphene but still weaker than with $\mathrm{C}_{60}$. Even when the first neighbor Li...Li distance in the armchair nanoribbon chosen here is slightly smaller than in the $4 \times 4$ graphene supercell, the Coulomb repulsion between ions is stronger in graphene. Li atoms in graphene have four first neighbors (two-dimensional structure) while nanoribbons only have one first-neighbor (one-dimensional structure). This difference increases the electrostatic repulsion between ions in graphene. As in the case of graphene, Li adsorption on armchair nanoribbons does not induce spin polarization in these systems.

There is a slightly stronger interaction between $\mathrm{Li}$ and armchair GNRs when the Li atom is on the hex-1 position that corresponds to the hexagons at the edges (Figure 2). In all other cases, there are no significant differences in the binding energies for the width studied here. For wider ribbons, we expect $E_{b}$ to tend asymptotically to a value somewhat larger than the one calculated in graphene due to their one-dimensional nature and its consequence on the number of first-neighbor $\mathrm{Li}$ atoms.

\section{Zigzag nanoribbons}

One of the most remarkable results found here is that the interaction of Li with zigzag GNRs is much stronger than with armchairs. As shown in Figure 2, the binding energy of Li at the edges of zigzag nanoribbons is more than $50 \%$ stronger than in graphene for both functionals, LSDA and PBE. This interaction slowly weakens as the adsorption position is shifted toward the center of the nanoribbon suggesting that narrow zigzag GNRs will be the most ideal to attain larger ion concentrations. While graphene and armchair nanoribbons do not develop magnetism upon $\mathrm{Li}$ adsorption, the charge transfer between the $\mathrm{Li}$ atom and the zigzag nanoribbon affects significantly its magnetic properties providing yet another interaction mechanism that is not present in graphene and armchair nanoribbons.

It is worth mentioning the effect of geometry relaxation in the binding energy for the different adsorption sites. We find that this effect is rather small across the hex -1 , hex -2 , hex -3 , and hex -4 series and accounts for, at most, $0.06 \mathrm{eV}$ of the total binding energy, calculated as the difference between the binding energies of the fully relaxed system and the rigid model approach at the equilibrium distance. This indicates that the stronger binding energy at the edges does not originate in deformations of the carbon backbone.

To further investigate the interaction of $\mathrm{Li}$ and zigzag 
nanoribbons we calculated the binding energies for the $h e x-i$ series with the hybrid HSE functional. We obtain binding energies of $1.55,1.27,1.16$, and $1.13 \mathrm{eV}$ for hex $-i$ with $i=1,2,3,4$, respectively. These results confirm the trend predicted by the LSDA and PBE functionals.

Zigzag edges exemplify the importance of edge effects in honeycomb lattices. 26, 49] Zigzag nanoribbons are expected to present a spin-polarized ground state characterized by an antiferromagnetic spin arrangement (AFM) with opposite spins at each edge. [50] The high spin state solution, with all spins ferromagnetically aligned (FM), is higher in energy than the AFM state by $10 \mathrm{meV} /$ edge atom for a $1.8 \mathrm{~nm}$ wide ribbon. This magnetic behavior has been predicted to present robustness with respect to some edge defects and impurities. [10, 26] However, we find that when $\mathrm{Li}$ is adsorbed on the zigzag nanoribbon, the charge that is transferred from the Li atom to the nanoribbon quenches the magnetization of the carbon atoms in the vicinity of the adsorption site in such a way that magnetization at both edges is no longer compensated, resulting in a net magnetic moment per cell that strongly depends on the adsorption position. Interestingly, except in the hex -1 case that quenches the magnetization at the edge in which the Li atom is adsorbed, the AFM and FM solutions become almost degenerated [51] while presenting significantly different total magnetic moments as shown in Table 1. These effects are shown in Figure 3 where the LSDA spin density maps $0.05 \AA$ above the surface of the AFM solution (ground state) of the pristine graphene nanoribbon and the different positions of the $\mathrm{Li}$ atom for the Li-GNR systems are presented for every adsorption site. We show the FM solution for the Li-GNR system as the AFM solution is very similar to the pristine ribbon but with a very small spin density in the vicinity of the adsorption site, just as in the FM case. In all cases, the Li adatom suppresses the spin polarization surrounding it, in such a way that spin-polarized edges do not appear for the hex-1 position on the Li side but magnetism slowly develops for hex-2 and increases for hex-3 and hex-4. It is worth mentioning at this point that a spin compensated calculation using the local density approximation produces a nonmagnetic metallic solution. This solution is higher in energy than the spin-polarized solution by about $70 \mathrm{meV} /$ cell for hex -1 and $108 \mathrm{meV} /$ cell for hex -4 . In the pristine case, the energy difference between the nonmagnetic and AFM solution is about $160 \mathrm{meV} /$ cell. Therefore, if we do not consider spin-polarization at all and perform all the calculations but the isolated $\mathrm{Li}$ atom using the spin-restricted LDA functional, the binding energy of the $\mathrm{Li}$ adatom in the different positions remains almost the same as in the spin-polarized case. These results indicate that edge morphology rather than magnetization is responsible for the enhanced interaction between $\mathrm{Li}$ and zigzag nanoribbons and a that a stronger binding is expected in zigzag nanoribbons regardless their magnetic nature.

In order to understand the difference in the adsorption

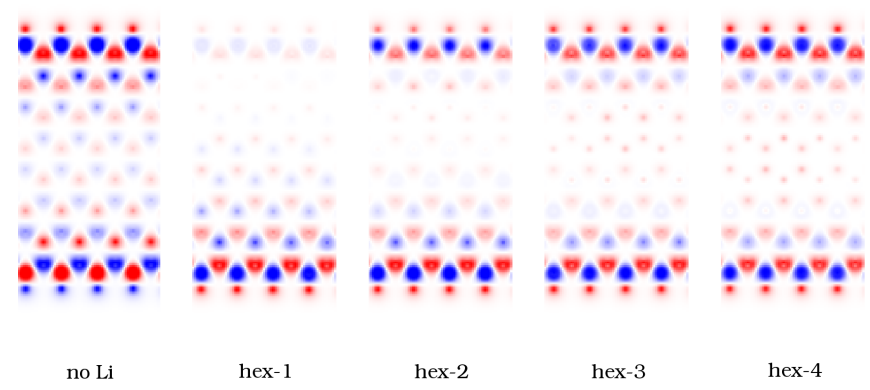

FIG. 3: LSDA spin density maps $0.05 \AA$ above the surface of the zigzag graphene nanoribbon for different positions of the $\mathrm{Li}$ atom. Blue and red colors represent opposite spin polarization.

strength of Li in zigzag and armchair nanoribbons, we analyze the total and partial density of states (DOS) in the isolated systems. In Figure 4 (b) we present the total DOS of a zigzag and armchair nanoribbons. The DOS corresponding to the zigzag nanoribbons is much larger for states close to the Fermi level $\left(\mathrm{E}_{F}\right)$ than in armchair ribbons. This larger number of states makes the charge transfer from the $\mathrm{Li}$ atom more accessible in the case of zigzag than in armchairs GNRs. Moreover, the stronger interaction at the edges of zigzag nanoribbons can also be understood by considering the partial DOS. In Figure 4 (c) and (d) we show the total and partial DOS for armchair (c) and zigzag (d) nanoribbons. The partial DOS is constructed by only considering $p$ orbitals perpendicular to the GNR from $\mathrm{C}$ atoms belonging to the different layers shown in Figure 4 (a). In this way, we can separate the contributions to the total DOS from the edges (layer 1) and the center part of the ribbon (layer 4). As seen in Figure 4 (c) and (d), while for the armchair GNR all layers contribute about the same to the total DOS in the vicinity of the Fermi level, the corresponding contributions in zigzag nanoribbons are significantly different. In the zigzag case, both, valence and conduction bands close to $\mathrm{E}_{F}$ are dominated by edge states (from layer 1) and layer 2 while layers 3 and 4 present significant smaller contributions. This explains why the binding energy of the adatom is much stronger at the edges and slowly decreases toward the center of the zigzag nanoribbon.

\section{CONCLUSIONS}

In summary, we have studied the adsorption of $\mathrm{Li}$ atoms at the hollow sites of fullerenes, graphene, and graphene nanoribbons (zigzag and armchair) by means of density functional theory within the local spin density and generalized gradient approximations. Li interacts with armchair nanoribbons and two-dimensional graphene through the same charge transfer mechanism, 
(a)

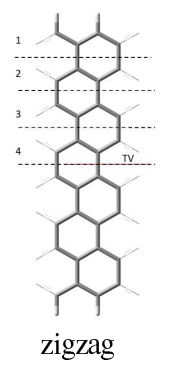

(c)

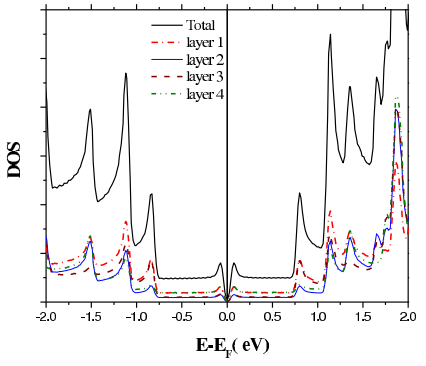

(b)

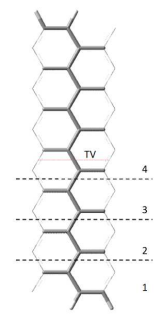

armchair

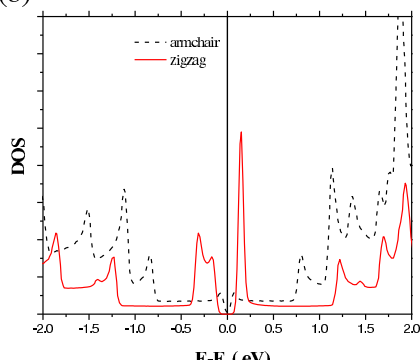

(d)

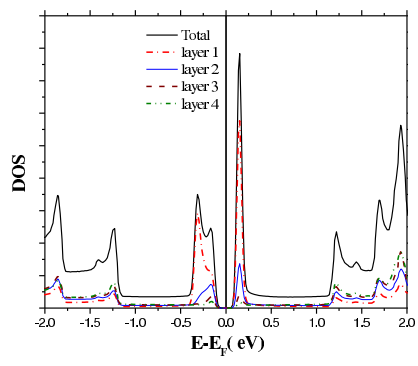

FIG. 4: (a) Schematic representation of the different $\mathrm{C}$ atom layers in zigzag and armchair nanoribbons. (b) Total DOS for the armchair and zigzag nanoribbon studied in this work. (c) Total and partial DOS for the armchair GNR with contributions from the 4 different layers shown in (a). (d) Total and partial DOS for the zigzag GNR with contributions from the 4 different layers shown in (a).

with binding energies per adatom of about $1.70 \mathrm{eV}$ and $1.55 \mathrm{eV}$ (LSDA) and $1.04 \mathrm{eV}$ and $1.20 \mathrm{eV}$ (PBE), respectively. Li interacts with zigzag nanoribbons in a much stronger way. The binding energy of Li at the edges of zigzag nanoribbons is $2.27 \mathrm{eV}$ (LSDA) and $1.70 \mathrm{eV}$ (PBE), more than 50\% stronger than in graphene. The binding energy progressively decreases as the adsorption position is shifted toward the center of the nanoribbon suggesting that narrow zigzag GNRs will be most ideal to attain larger ion concentrations.

While the charge transfer between the Li adatom and the zigzag nanoribbon affects significantly the magnetic properties of the latter providing an additional interaction mechanism that is not present in graphene or armchair nanoribbons, we find that the morphology of the edges, rather than magnetization, is responsible for the enhanced $\mathrm{Li}$ adsorption. These results illustrate the importance of controlling the edges of GNRs with atomic precision in order to maximize their potential for technological applications. This precision has been demonstrated to be experimentally achievable. 52.

\section{Acknowledgments}

Acknowledgment is made to the donors of The American Chemical Society Petroleum Research Fund for support of this research through the award ACS PRF \#49427-UNI6. The authors would like to acknowledge the useful comments of the anonymous referee.
[1] C. Berger, Z. Song, X. Li, X. Wu, N. Brown, C. Naud, D. Mayou, T. Li, J. Hass, A. N. Marchenkov, et al., Science 312, 1191 (2006).

[2] D. Abanin and L. S. Levitov, Science 317, 641 (2007).

[3] M. Fujita, K. Wakabayashi, K. Nakada, and K. Kusakabe, J. Phys. Soc. Jpn. 65, 1920 (1996).

[4] L. Pisani, J. Chan, B. Montanari, and N. M. Harrison, Phys. Rev. B 75, 064418 (2007).

[5] K. Wakabayashi, M. Sigrist, and M. Fujita, J. Phys. Soc. Jpn. 67, 2089 (1998).

[6] K. Wakabayashi, M. Fujita, H. Ajiki, and M. Sigrist, Phys. Rev. B 59, 8271 (1999).

[7] K. Kusakabe and M. Maruyama, Phys. Rev. B 67, 092406 (2003).

[8] A. Yamashiro, Y. Shimoi, K. Harigaya, and K. Wakabayashi, Phys. Rev. B 68, 193410 (2003).

[9] H. Lee, Y.-W. Son, N. Park, S. Han, and J. Yu, Phys. Rev. B 72, 174431 (2005).

[10] Y.-W. Son, M. Cohen, and S. Louie, Nature 444, 347 (2006).
[11] H. Hsu and L. Reichl, Phys. Rev. B 76, 045418 (2007).

[12] P. Shemella, Y. Zhang, M. Mailman, P. Ajayan, and S. Nayak, Appl. Phys. Lett. 91, 042101 (2007).

[13] Y. Zhang, Y.-W. Tan, H. L. Stormer, and P. Kim, Nature 438, 201 (2005).

[14] N. M. R. Peres, A. H. Castro-Neto, and F. Guinea, Phys. Rev. B 73, 195411 (2006).

[15] N. M. R. Peres, A. H. Castro-Neto, and F. Guinea, Phys. Rev. B 73, 241403 (2006).

[16] K. S. Novoselov, Z. Jiang, Y. Zhang, S. V. Morozov, H. L. Stormer, U. Zeitler, J. C. Maan, G. S. Boebinger, P. Kim, and A. K. Geim, Science 315, 1379 (2007).

[17] L. Brey and H. A. Fertig, Phys. Rev. B 73, 235411 (2006).

[18] M. Ezawa, Phys. Rev. B 73, 045432 (2006).

[19] V. Barone, O. Hod, and G. E. Scuseria, Nano Lett. 6, 2748 (2006).

[20] M. Y. Han, B. Ozyilmaz, Y. Zhang, and P. Kim, Phys. Rev. Lett. 98, 206805 (2007).

[21] C. T. White, J. Li, D. Gunlycke, and J. W. Mintmire, Nano Lett. 7, 825 (2007). 
[22] L. Brey and H. A. Fertig, Phys. Rev. B 75, 125434 (2007).

[23] P. G. Silvestrov and K. B. Efetov, Phys. Rev. Lett. 98, 016802 (2007).

[24] D. Gunlycke, D. Areshkin, and C. White, Appl. Phys. Lett. 90, 142104 (2007).

[25] B. Obradovic, R. Kotlyar, F. Heinz, P. Matagne, T. Rakshit, M. D. Giles, M. A. Stettler, and D. E. Nikonov, Appl. Phys. Lett. 88, 142102 (2006).

[26] O. Hod, V. Barone, J. E. Peralta, and G. Scuseria, Nano Lett. 7, 2295 (2007).

[27] D. E. Jiang, B. G. Sumpter, and S. Dai, J. Chem. Phys. 126, 134701 (2007).

[28] A. Ferre-Vilaplana, J. Phys. Chem. C 112, 3998 (2008).

[29] J. I. Martínez, I. Cabria, M. J. López, and J. A. Alonso, J. Phys. Chem. C 113, 939 (2008).

[30] E. Yoo, J. Kim, E. Hosono, H. Zhou, T. Kudo, and I. Honma, Nano Lett. 8, 2277 (2008).

[31] W.-Q. Deng, X. Xu, and W. Goddard, Phys. ReV. Lett. 92, 166103 (2004).

[32] Q. Sun, P. Jena, Q. Wang, and M. Marquez, J. Am. Chem. Soc. 128, 9741 (2006).

[33] G. Mpourmpakis, E. Tylianakis, and G. E.Froudakis, Nano Lett. 7, 1893 (2007).

[34] C. Ataca, E. Akturk, S. Ciraci, and H. Ustune, Appl. Phys. Lett. 93, 043123 (2008).

[35] M. J. Frisch, G. W. Trucks, H. B. Schlegel, G. E. Scuseria, M. A. Robb, J. R. Cheeseman, J. A. Montgomery, Jr., T. Vreven, K. N. Kudin, et al., Gaussian Development Version (Rev. f.1) (Gaussian Inc., Pittsburgh, PA, 2008).

[36] J. Slater, Quantum Theory of Molecular and Solids, The Self-Consistent Field for Molecular and Solids, Vol. 4 (McGraw-Hill, NewYork, 1974).
[37] S. H. Vosko, L. Wilk, and M. Nusair, Can. J. Phys. 58, 1200 (1980).

[38] J. P. Perdew, K. Burke, and M. Ernzerhof, Phys. Rev. Lett. 77, 3865 (1996).

[39] J. P. Perdew, K. Burke, and M. Ernzerhof, Phys. Rev. Lett. 78, 1396 (1997).

[40] This basis set consists of (3s2p1d) contracted Gaussian functions for $\mathrm{C}$ and $\mathrm{Li}$ and $(2 \mathrm{~s} 1 \mathrm{p})$ for $\mathrm{H}$.

[41] J. Heyd, G. E. Scuseria, and M. Ernzerhof, J. Comp. Phys. 118, 8207 (2003).

[42] J. Heyd, G. E. Scuseria, and M. Ernzerhof, J. Comp. Phys. 124, 219924(E) (2006).

[43] J. Heyd and G. E. Scuseria, J. Comp. Phys. 120, 7274 (2004).

[44] J. Heyd and G. E. Scuseria, J. Comp. Phys. 121, 1187 (2004).

[45] F. Valencia, A. Romero, F. Ancilotto, and P. Silvestrelli, J. Phys. Chem. B 110, 14832 (2006).

[46] K. Chan, J. Neaton, and M. Cohen, Phys. Rev. B 77, 235430 (2008).

[47] S. F. Boys and F. Bernardi, Mol. Phys. 19, 553 (1970).

[48] M. Khantha, N. A. Cordero, L. M. Molina, J. A. Alonso, and L. A. Girifalco, Phys. Rev. B 70, 125422 (2004).

[49] V. Barone and J. E. Peralta, Nano Lett. 8, 2210 (2008).

[50] K. Nakada, M. Igami, and M. Fujita, J. Phys. Soc. Jpn. 67, 2388 (1998).

[51] The FM state becomes actually more stable than the AFM by about $4 \mathrm{meV}$.

[52] S. S. Datta, D. R. Strachan, S. M. Khamis, and A. T. Johnson, Nano Lett. 8, 1912 (2008). 